Revista de Psicología Vol. 39 (2), 2021 (e-ISSN 2223-3733)

\title{
Adolescentes chilenos ante la inmigración latinoamericana: perfiles aculturativos, prejuicio, autoeficacia cultural y bienestar $^{1}$
}

\author{
María José Mera-Lemp ${ }^{2}$, Gonzalo Martínez-Zelaya ${ }^{3}$, Marian Bilbao ${ }^{4}$ \\ Universidad Alberto Hurtado - Chile ${ }^{1,3}$, Universidad Viña del Mar-Chile ${ }^{2}$
}

El nuevo escenario sociocultural producido por la inmigración en los colegios implica un complejo proceso de ajuste psicosocial. Los estudiantes nacionales también deben enfrentar diversos y nuevos desafíos producto de la inmigración, que se vinculan tanto con la percepción y gestión de las diferencias culturales. Este trabajo tiene como objetivo identificar perfiles aculturativos en una muestra de estudiantes secundarios chilenos $(N=426)$. $\mathrm{El}$ análisis de conglomerados permitió encontrar una solución de tres perfiles aculturativos (Individualismo Tolerante, Individualista Indiferenciado y Etnocentrista Indiferenciado). Los resultados muestran que dichos perfiles se diferencian en Prejuicio Afectivo, Autoeficacia Cultural y Bienestar. Se discute sobre la importancia de generar entornos que favorezcan el contacto intergrupal positivo, para así posibilitar el desarrollo de la competencia intercultural desde etapas tempranas de la vida.

Palabras clave: Aculturación, sociedad receptora, prejuicio, autoeficacia cultural, bienestar.

Chilean teenagers faced with Latin American immigration: acculturative profiles, prejudice, cultural self-efficacy and well-being

The new socio-cultural scenario produced by immigration in schools implies a complex process of psychosocial adjustment. National students must also face diverse and new challenges as a result of immigration, which are linked to the perception and management of cultural differences. This paper aims to identify acculturative profiles in a sample $(N=426)$

1 Este trabajo contó con el financiamiento, para la primera autora, del proyecto CONICYT / FONDECYT Postdoctoral $N^{\circ} 3180774$, y de Laureate Center for Youth Studies at Universidad Andres Bello, Chile.

2 Doctora en Psicología Social. Investigadora posdoctoral, Facultad de Psicología. Dirección Postal: Almirante Barroso 26, Santiago de Chile, Chile. CP: 8340575 Contacto: mariajosemera@yahoo.es https://orcid.org/0000-0001-5763-6913

3 Doctor en Psicología Social. Académico Universidad Viña del Mar, Chile. Dirección postal: Los Fresnos 91, Miraflores, Viña del Mar, Chile. CP: 2561091. Contacto: gonzalo.martinez@uvm.cl https://orcid.org/0000-0002-9848-3666.

4 Doctora en Psicología Social. Profesora adjunta, Facultad de Psicología. Dirección postal: Almirante Barroso 26, Santiago de Chile, Chile. CP: 8340575. Contacto: mbilbao@uahurtado.cl https://orcid.org/0000-0002-5984-4908 
of Chilean secondary school students. Cluster analysis allowed us to find a solution of three acculturative profiles (Tolerant Individualist, Undifferentiated Individualist and Undifferentiated Ethnocentric). Results show that these profiles differ in their levels of Affective Prejudice, Cultural Self-efficacy, and Well-being. These findings stress the importance of promoting social environments which could improve positive intergroup contact to enable the development of intercultural competence from early stages of life. Keywords: Acculturation, Host society, Prejudice, Cultural self-efficacy, Wellbeing.

Adolescentes chilenos diante da imigraçáo latino-americana: perfis aculturativos, preconceito, autoeficácia cultural e bem-estar

O novo cenário sociocultural produzido pela imigração nas escolas implica um complexo processo de ajuste psicossocial. Os estudantes nacionais também devem enfrentar diversos e novos desafios decorrentes da imigraçáo, que estão ligados tanto à percepçáo quanto à gestão das diferenças culturais. Este trabalho tem como objetivo identificar perfis aculturativos em uma amostra de estudantes chilenos do ensino médio $(n=426)$. A análise de cluster permitiu encontrar uma solução de três perfis aculturativos (Individualismo Tolerante, Individualista Indiferenciado e Etnocêntrico Indiferenciado). Os resultados mostram que esses perfis diferem em Preconceito Afetivo, Autoeficácia Cultural e Bem-estar. Discute-se a importância de criar ambientes que favoreçam o contato intergrupal positivo, a fim de possibilitar o desenvolvimento da competência intercultural desde as primeiras fases da vida. Palavras-chave: Aculturação, Sociedade receptora, Prejuízo, Autoeficácia cultural, Bem estar.

Les adolescents chiliens face à l'immigration latino-américaine : profils d'acculturation, les préjugés, l'auto-efficacité culturelle et le bien-être

Le nouveau scénario socioculturel produit par l'immigration dans les écoles implique un processus complexe d'ajustement psychosocial. Les étudiants nationaux doivent également faire face à des défis divers et nouveaux résultant de l'immigration, qui sont liés à la fois à la perception et à la gestion des différences culturelles. Ce travail vise à identifier des profils d'acculturation dans un échantillon de lycéens chiliens $(n=426)$. L'analyse de cluster a permis de trouver une solution de trois profils acculturatifs (l'individualisme tolérant, l'individualiste indifférencié et l'ethnocentrique indifférencié). Les résultats montrent que ces profils different en termes de préjugés affectifs, d'auto-efficacité culturelle et de bien-être. L'importance de créer des environnements favorisant des contacts intergroupes positifs est discutée, afin de permettre le développement de la compétence interculturelle dès les premières étapes de la vie.

Mots-clés: Acculturation, Société réceptrice, Préjudice, Auto-efficacité culturelle, Le bien-être. 
La sociedad chilena se ha visto tensionada, producto de la incorporación de un nuevo sujeto social en la vida cotidiana. El rápido aumento de la población inmigrante asentada en Chile tiene importantes repercusiones en el sistema educativo. Si en el año 2010 los extranjeros representaban el 1.8\% del total de habitantes del país, en el 2017 esta población alcanzó el 4.8\% (Instituto Nacional de Estadísticas, 2018), y en el ańo 2019 en 1.492.522 las personas extranjeras que viven en Chile de forma regular (INE, 2020), lo cual representa el $7.7 \%$ de la población, porcentaje más alto registrado en los últimos 100 ańos. Estos nuevos habitantes provienen principalmente de América Latina y el Caribe (90.96\%), tales como Venezuela (30.52\%), Perú (15.76\%), Haití (12.45\%), Colombia (10.80\%) y Bolivia (8.05\%) (INE, 2020).

Estos nuevos flujos migratorios, se caracterizan además por la creciente presencia de niños y adolescentes en edad escolar, que en 2018 eran el $15 \%$ de la población inmigrante (Instituto Nacional de Estadísticas, 2018). Esto ha tenido como efecto que la matrícula extranjera en el sistema educativo local se duplicara entre 2015 y 2017 (Ministerio de Educación de Chile, 2018). Aunque todavía es baja (2.2\%) en comparación con otros países receptores de inmigrantes de la OCDE (Fernández, 2018), este aumento ha tensionado al sistema educacional en múltiples niveles (Barrios-Valenzuela y Palou-Julián, 2014). Además, se ha observado que este nuevo alumnado tiende concentrarse en centros públicos (56\%) y en escuelas subsidiadas por el Estado (36\%), que atienden a la población de menores ingresos y con mayores índices de vulnerabilidad social del país (Joiko \& Vázquez, 2016).

El nuevo escenario social y cultural producido por la inmigración en los colegios implica un complejo proceso de ajuste psicosocial, tanto para los estudiantes inmigrantes como para los de la sociedad receptora (Salas et al., 2017). De hecho, los estudios que indagan en las experiencias de niños y adolescentes inmigrantes latinoamericanos en Chile han 
evidenciado dinámicas de discriminación y racialización que dificultan su integración social y cultural en la vida cotidiana (Arellano, Sanhueza, García, Muñoz, \& Norambuena, 2016; de la Torre, 2011; Hein, 2012; Pavez, 2012; Riedemann \& Stefoni, 2015; Tijoux, 2013).

Por su parte, los estudiantes miembros de la sociedad mayoritaria también deben enfrentar diversos y nuevos desafíos producto de la inmigración, que se vinculan tanto con la percepción y gestión de las diferencias culturales (Briones at al., 2008), como con el determinar de qué modo desean que los inmigrantes se incorporen, en términos sociales y culturales, al contexto de recepción (Bourhis, Moïse, Perreault \& Senécal, 1997; Sirlopú, Melipillán, Sánchez, \& Valdés, 2015; Zlobina \& Páez, 2018).

En el caso de los niños y adolescentes de las sociedades receptoras, la inmigración moviliza procesos sociocognitivos especialmente relevantes, puesto que se encuentran en una etapa del desarrollo donde las pertenencias grupales, el contacto y la comparación con otros diferentes, son elementos clave para construcción de sus identidades (Mera, Martínez-Taboada \& Costalat-Founeau, 2014; Migliorini, Rania \& Cardinale, 2014).

Además, durante la transición a la adolescencia se van desarrollando las capacidades para comprender el funcionamiento, el estatus y las normas grupales así como para entender la importancia de su mantención y estabilidad (Rutland \& Killen, 2015). El uso de estas habilidades sociocognitivas para construir conocimientos sobre contextos intergrupales, es determinante para el desarrollo y la expresión de prejuicios y sesgos hacia miembros de exogrupos, como serían las personas inmigrantes (Rutland \& Killen, 2015; Rania, Cardinali, Cifatte \& Migliorini, 2012; Vekuyten, Thijs \& Sierksma, 2015).

En Chile, las investigaciones que informan acerca de las actitudes de niños y adolescentes chilenos hacia la inmigración son escasas. En general, se trata de estudios dirigidos a explorar en los procesos de escolarización de alumnos inmigrantes, en los que la perspectiva de los estudiantes locales es analizada principalmente como correlato de estas experiencias, con algunas excepciones (e.g. Cárdenas, 2006; González \& 
Brown, 2017). Estos estudios, casi en su totalidad de carácter cualitativo (de la Torre, 2011; Pavez, 2012; Riedemann \& Stefoni, 2015; Salas et al., 2017; Tijoux, 2013), dan cuenta del desarrollo de actitudes negativas y conductas discriminatorias hacia los inmigrantes latinoamericanos, que han sido asociadas tanto al racismo, como a la dificultad para gestionar las diferencias culturales, dando origen a nuevas formas de exclusión social.

Desde una perspectiva psicosocial, estos cambios identitarios, actitudinales y conductuales bidireccionales, movilizados por el contacto directo o indirecto entre los miembros de las sociedades receptoras y de la población inmigrante, han sido conceptualizados como procesos de aculturación (Berry, 2005). El Modelo Interactivo de Aculturación, propuesto por Bourhis et al. (1997), propone que las formas en que los inmigrantes puedan participar en la sociedad receptora serán delimitadas por el grado en que los miembros de la cultura mayoritaria acepten que los nuevos habitantes mantengan sus identidades culturales de origen, y al mismo tiempo, les permitan incorporar elementos de la cultura dominante.

Berry (1997) diferencia cuatro orientaciones de aculturación: integracionismo, asimilacionismo, segregacionismo y exclusionismo. El integracionismo se produce cuando se valora positivamente la mantención de las culturas de origen, y al mismo tiempo, la adopción de aspectos de la cultura mayoritaria. El asimilacionismo ocurre cuando se desea la adopción de la cultura del grupo mayoritario y el abandono de las identidades culturales propias, mientras que el segregacionismo implica la conservación de la cultura de origen al margen de la sociedad receptora. El exclusionismo se define como el rechazo tanto de mantención de la cultura de origen como de la adopción de la cultura local por parte de los grupos inmigrantes.

Bourhis y colaboradores (1997) introducen una quinta alternativa, el individualismo, que tiene lugar cuando los sujetos se definen a sí mismos y a los otros no como miembros de categorías grupales, sino como individuos, centrando su atención en características y aspiraciones personales, y relevando la movilidad social por sobre la incorporación de la nueva cultura o la mantención de la propia. 
Las actitudes de aculturación son desarrolladas por miembros de las sociedades receptoras en la interacción con la población inmigrante, o previo a su encuentro, a partir del conocimiento social compartido con respecto a ella. Por esto, aunque ciertas preferencias aculturativas pueden tener un mayor predominio, su despliegue en la vida cotidiana es dinámico e interrelacionado, pudiendo por ejemplo variar en función del contexto (Barrette, Bourhis, Personnaz, \& Personnaz, 2004)

Las investigaciones realizadas con muestras transculturales y en diferentes países (Barrette et al., 2004; Berry et al., 2006; Bourhis et al., 2009) muestran que la mayoría de los grupos prefieren la integración, mientras que el exclusionismo es menos deseado (Berry et al., 2006). Las orientaciones de asimilación y segregación parecen ser más sensibles a las condiciones históricas y sociales del contexto en el que se produce la recepción de inmigrantes (Bourhis et al, 1997).

En Chile, los estudios que han indagado en las actitudes de los miembros de la sociedad mayoritaria hacia el intercambio cultural con inmigrantes latinoamericanos han focalizado en muestras de adultos. Estas investigaciones (Mera, Martínez-Zelaya, Bilbao \& Garrido, 2017; Sirlopú, Melipillán, Sánchez \& Valdés, 2015), han identificado al individualismo como estilo de aculturación predominante, que favorecería la participación e inclusión de los inmigrantes debido a su mayor adecuación a los modelos de ciudadanía promovidos por la ideología neoliberal en el país durante las últimas décadas.

Los estudios que examinan las preferencias aculturativas de miembros de sociedades receptoras ponen de relevancia su relación con el prejuicio, entendido como una actitud o respuesta emocional negativa hacia un exogrupo social o sus miembros (Dovidio, Hewstone, Glick, \& Esses, 2010; Rojas, Navas, Sayans-Jimenez \& Cuadrado, 2014). El prejuicio es determinado por los efectos de las interacciones sociales en el estatus y el poder de los individuos (Kemper, 1984), y permite reforzar las identidades grupales, determinando el tipo de contacto que se sostiene con los miembros de grupos a los que no se pertenece (Aberson, 2015; Keltner \& Haidt, 1999). 
Si bien la dimensión afectiva del prejuicio ha sido tradicionalmente vinculada con la expresión directa de emociones negativas de rechazo, odio y hostilidad, se ha establecido que otros sentimientos negativos más sutiles o indirectos, como la percepción de incomodidad o inseguridad ante el exogrupo (Navas \& Rojas, 2010) también inciden en la construcción y estabilidad de relaciones de poder desiguales entre los grupos (Dovidio et al., 2010; Yzerbyt \& Demoulin, 2010).Además, se considera que otro relevante indicador de prejuicio está dado por la ausencia de emociones positivas ante otros grupos sociales y sus miembros (Pettigrew \& Meertens, 1995).

En general, los estudios que profundizan en la asociación entre prejuicio y aculturación, han mostrado que altos niveles de prejuicio se vinculan con la oposición a que los inmigrantes mantengan su identidad cultural de origen, relacionándose con el exclusionismo y el asimilacionismo (Brown \& Zagefka, 2011; Navas, García, Rojas, Pumares, \& Cuadrado, 2006; Navas \& Rojas, 2010; Zagefka et al., 2012).

En cambio, bajos niveles de prejuicio se han asociado con una menor preocupación por que los inmigrantes mantengan su propia cultura, pudiendo relacionarse tanto con la integración, como con la separación (Brown y Zagefka, 2011; Navas et al., 2006; Navas \& Rojas, 2010; Zagefka et al., 2012). Estos resultados han sido observados también en muestras de adolescentes de países receptores de inmigrantes (Briones, Verkuyten, Cosano \& Tabernero, 2012).

Por otro lado, algunas investigaciones han vinculado las actitudes hacia miembros de grupos minoritarios con el desarrollo de competencias interculturales (Early \& Ang, 2003; Li \& Gasser, 2005; Rania, Cardinali, Cifatte \& Migliorini, 2012; Rockstuhl \& Ng, 2008), definidas como la habilidad para pensar y actuar de manera exitosa en contextos interculturales (Hammer, Bennet \& Wiesemann, 2003). Estas competencias se asocian con conocimientos, capacidades y actitudes que permiten relacionarse adecuadamente con personas de diferentes orígenes nacionales y culturales (Johnson, Lenartowicz \& Apud, 2006).

Algunos autores (Briones et al., 2009; Fan \& Mak, 1998; Li \& Gasser, 2005; Rania et al., 2012), han enfatizado la relevancia de la 
percepción que los individuos tienen sobre su propia competencia intercultural para relacionarse exitosamente en contextos de diversidad cultural. Este sentimiento de eficacia personal tiene una importante incidencia en la adaptación y el afrontamiento de los cambios, e influye en los procesos cognitivos, motivaciones, emocionales y decisionales de las personas (Rania et al., 2012). Se ha observado que el incremento de la percepción de autoeficacia cultural se vincula con la presencia de expectativas positivas ante el contacto con miembros de otros grupos culturales, mejorando la calidad de las interacciones intergrupales. Además, la autoeficacia cultural atenúa la percepción de ansiedad, y se asocia con una mayor satisfacción con el contacto intercultural. De esta manera, el sentimiento de eficacia cultural tiende a motivar a la interacción, incluso en circunstancias en que no se cuente con todos los recursos culturales necesarios para este intercambio (Briones et al., 2009; Briones, 2010).

Desde este enfoque, algunos estudios (Li \& Gasser, 2005; Rania et al., 2012; Rockstuhl \& Ng, 2008; Rodenborg \& Boisen, 2013; Spencer-Rodgers \& McGovern, 2002; Ting-Toomey, 2009), han dado cuenta de que en la medida en que los individuos se perciben a sí mismos como interculturalmente competentes, su contacto con miembros de otros grupos sociales es de mejor calidad y, de esta manera, presentan una mayor preferencia por su integración. Por el contrario, se ha observado que mientras menor es la percepción de competencia intercultural, mayor es la preferencia por la exclusión y la asimilación (Briones et al., 2009). Esta línea de investigación ha generado un creciente interés en las últimas décadas debido a la relevancia de identificar variables que faciliten reducir las actitudes intergrupales negativas en contextos interculturales y en especial en escenarios altamente sensibles como son la escuela, los servicios sociales y las instituciones de salud (Chen \& Starosta, 1998; Earley \& Ang 2003; Hammer et al., 2003; Rodenborg \& Boisen, 2013; Seeberg \& Minick, 2012; Leung, Ang \& Tan, 2014).

Otra línea de investigación emergente en este ámbito es la que indaga en el bienestar percibido, en términos del funcionamiento 
psicológico y social positivo, como correlato de las actitudes de miembros de grupos mayoritarios hacia los inmigrantes. La evidencia disponible (Migliorini, Rania \& Cardinali, 2016) indica que existen relaciones positivas entre el bienestar y el deseo de que los inmigrantes se integren a la sociedad, mientras que su relación con el exclusionismo es inversa (Migliorini et al., 2016). Así también, un estudio con población chilena adulta reportó que los sujetos con perfil aculturativo individualista percibían mayor bienestar que los integracionistas y exclusionistas, sugiriendo que en el contexto sociocultural chileno, la adhesión al individualismo puede resultar más adaptativa para la población local (Mera et al., 2017).

En vista de los antecedentes presentados y la escasa evidencia que existe en población adolescente de sociedades receptoras, este trabajo tiene como objetivo identificar perfiles aculturativos en una muestra de estudiantes secundarios chilenos. Además, busca establecer la existencia de diferencias entre perfiles aculturativos en cuanto al prejuicio percibido, la autoeficacia cultural y el bienestar. Con base en la literatura, se espera que el predominio del integracionismo y el individualismo se asocie con un menor grado de prejuicio, mayor autoeficacia cultural y mayor bienestar, que en grupos en los que predominen las demás orientaciones de aculturación.

\section{Método}

\section{Participantes}

La muestra estuvo compuesta por 426 escolares chilenos, pertenecientes a cuatro centros que imparten Educación Secundaria. El 49.9\% fueron mujeres, con edades entre 14 y 19 años $(M=15.77 ; D E=1.162)$. El $86.4 \%$ de los participantes estudiaba en establecimientos educativos públicos, mientras que el $13.6 \%$ asistía a centros subsidiados por el Estado que principalmente a población de nivel socioeconómico bajo. El 77\% de los participantes no tenía compańeros de aula inmigrantes, mientras que el $23 \%$ tenía entre 1 y 3 compañeros inmigrantes latinoamericanos. 


\section{Medición}

Orientaciones de Aculturación: se utilizó la sub escala de "Mantención cultural", de la Escala de Aculturación para la sociedad receptora (Montreuil y Bouhris, 2001), adaptada a Chile por Sirlopú, Melipillán, Sánchez y Valdés (2015). La escala consta de 5 ítems tipo Likert con 7 opciones de respuesta ( $1=$ totalmente en desacuerdo, $7=$ totalmente de acuerdo), que refieren al grado en que los sujetos desean la integración de los inmigrantes ("Los inmigrantes latinoamericanos que viven en Chile deberían mantener su cultura y a la vez adoptar la cultura chilena"), segregación ("Los inmigrantes latinoamericanos que viven en Chile deberían mantener su cultura de origen, pero no debiesen mezclarla con la cultura chilena"), asimilación ("Los inmigrantes latinoamericanos que viven en Chile deberían renunciara su cultura de origen y adoptar la cultura chilena"), exclusión ("Los inmigrantes latinoamericanos que viven en Chile no deberían mantener su cultura y tampoco adoptar la cultura chilena, porque, en cualquier caso, debería haber menos inmigración a este país") e individualismo ("El que los inmigrantes latinoamericanos que viven en Chile mantengan su cultura de origen o adopten la cultura chilena no hace diferencias, porque cada persona es libre de adoptar la cultura de su elección”).

Prejuicio afectivo: Se aplicó la escala de emociones de Navas y Rojas (2010), que está compuesta por 11 ítems tipo Likert con 5 opciones de respuesta $(1=$ totalmente en desacuerdo, $5=$ totalmente de acuerdo $)$, donde 3 ítems miden emociones positivas (respeto, admiración, simpatía), 4 ítems evalúan emociones negativas sutiles (desconfianza, incomodidad, inseguridad, indiferencia), y 4 ítems refieren a emociones negativas tradicionales (odio, desprecio, irritación, miedo). La fiabilidad para esta escala fue de $\alpha_{\text {Cronbach }}=.861 \mathrm{y} \omega_{\text {McDonald }}=.866$. Respecto de la estructura, el modelo se ajusta de la siguiente forma: $\chi^{2}(32, N=426)=88.772$, $p<.001 ; \mathrm{CFI}=.980 ; \mathrm{TLI}=.965 ; \mathrm{NNFI}=.686 ; \mathrm{PNFI}=.564$; SRMR $=.034 ;$ RMSEA $=.065$ (90\% CI [.049, .081]).

Autoeficacia Cultural: se utilizó la Escala de Autoeficacia Cultural para Adolescentes (Briones et al., 2009). La escala original cuenta con 
25 ítems tipo Likert con 5 opciones de respuesta $(1=$ totalmente en desacuerdo, 5= totalmente de acuerdo) que miden cinco dimensiones: autoeficacia para procesar información proveniente de otras culturas, relacionarse satisfactoriamente con otras culturas, comprender diferentes estilos de vida, el afrontamiento de la soledad y añoranza, y en el aprendizaje y comprensión de nuevas lenguas. Debido a que en este estudio se trabajó con una muestra compuesta por estudiantes autóctonos, se aplicaron 18 ítems $\left(\alpha_{\text {Cronbach }}=.88 ; \omega_{\text {McDonald }}=.90\right)$ que corresponden a las dimensiones de autoeficacia para procesar información de otras culturas $(\alpha=.82)$ ("Cuando hablo con personas de otra cultura, soy capaz de hacerme entender al hablar”), relacionarse con otras culturas $(\alpha=.89)$ ("Al relacionarme con personas de otra cultura, soy capaz de disfrutar de actividades propias de la gente de esa cultura"), y comprender otros estilos de vida $(\alpha=.85)$ ("Al aproximarme a otra cultura, soy capaz de entender la forma en que se relacionan las parejas de otra cultura"). Respecto de la estructura, el modelo se ajusta de la siguiente forma: $\chi^{2}(120, N=426)=284.071, p<.001$; $\mathrm{CFI}=.966 ; \mathrm{TLI}=.956 ; \mathrm{NNFI}=.956 ; \mathrm{PNFI}=.739 ; \mathrm{SRMR}=.034$; RMSEA $=.057(90 \%$ CI $[.048, .065])$.

Bienestar: se aplicó la sub escala de bienestar recordado del Índice de Felicidad de Pemberton (PHI) de Hervas y Vázquez (2013). Este instrumento consta de 11 ítems tipo Likert relacionados con distintos ámbitos del recuerdo de bienestar general ("Me siento muy satisfecho/a con mi vida"), eudaimónico ("Mi vida está llena de aprendizajes y desafíos que me hacen crecer"), hedonista ("Disfruto cada día de muchas pequeńas cosas"), y el bienestar social ("Siento que vivo en una sociedad que me permite desarrollarme plenamente"), con un rango de respuesta de 0 (totalmente en desacuerdo) a 10 (totalmente de acuerdo). En la muestra estudiada en esta investigación, la escala posee una fiabilidad general de $\alpha_{\text {Cronbach }}=.89 \mathrm{y} \omega_{\text {McDonald }}=.90$. Respecto de la estructura, el modelo se ajusta de la siguiente forma: $\chi^{2}(38$, $N=426)=89.211, p<.001 ; \mathrm{CFI}=.978 ; \mathrm{TLI}=.967 ; \mathrm{NNFI}=.967$; PNFI $=.665 ;$ SRMR $=.029$; RMSEA $=.056(90 \%$ CI $[.041, .071])$. 


\section{Procedimiento}

Se realizó un muestreo por conveniencia de acuerdo a la disponibilidad de los centros educativos para participar en el estudio. En total, cuatro centros educativos autorizaron la participación de sus estudiantes en la investigación. Los cuestionarios fueron aplicados por miembros del equipo de investigación en los establecimientos educativos y durante el horario de clases. La modalidad utilizada fue de tipo autoadministrada. El tiempo de aplicación fue de aproximadamente 45 minutos.

El proyecto y los consentimientos informados fueron sometidos a la evaluación del Comité de Ética de la Universidad Alberto Hurtado, y se siguieron todos los principios éticos de la Declaración de Helsinki para investigación con seres humanos. Previo a la aplicación de los cuestionarios, se solicitó a los directores de los establecimientos educativos la firma de una carta en la que autorizaban la realización de la investigación en sus centros. Posteriormente, se solicitó a padres y tutores de los alumnos la firma de un documento de consentimiento informado en el que los autorizaban a responder un cuestionario. Luego, se entregó a los estudiantes menores de edad un documento de asentimiento, y a aquellos que habían cumplido la mayoría de edad, un documento de consentimiento informado que debían firmar si deseaban participar del estudio y donde se aseguraba la confidencialidad y anonimato de la información producida. Todos los encuestados participaron de manera voluntaria y fueron debidamente informados acerca del objetivo y las características de este estudio. La información fue tratada de manera confidencial y se eliminó cualquier elemento que pudiera dar cuenta de la identidad de los participantes.

\section{Análisis de datos}

Se realizaron análisis factoriales confirmatorios para determinar la validez de los instrumentos utilizados, con el programa JASP V.9.9. Posteriormente, se realizaron comparaciones de medias con el fin de detectar la posible influencia del contacto cotidiano con compañeros 
de aula inmigrantes. Debido a que no se encontraron diferencias estadísticamente significativas entre estudiantes con compañeros inmigrantes en aula y estudiantes sin compañeros inmigrantes en aula, para efectos de los análisis posteriores se consideró a ambos grupos como una misma muestra (Ver Tabla 1). Luego, se hicieron análisis de clusters con el fin de identificar grupos de sujetos basados en diferencias en sus puntajes en las preferencias de aculturación. Así, los sujetos cuyos puntajes mostraron patrones similares a través de las variables fueron agrupados y diferenciados de otros grupos que mostraban patrones diferentes en sus puntajes (Mooi y Sarstedt, 2011). Por último, se realizaron análisis de varianza (ANOVA) y pruebas post hoc de Tamhane para detectar la existencia de diferencias estadísticamente significativas entre los grupos, en los puntajes obtenidos en prejuicio afectivo, autoeficacia cultural, y bienestar.

\section{Resultados}

Los resultados obtenidos muestran que el individualismo fue la preferencia aculturativa más respaldada por los participantes, con una media de $5.49(D E=1.64)$ en una escala de 7 puntos, seguida por el integracionismo $(M=5.09 ; D E=1.74)$, y el segregacionismo $(M=4.69$; $D E=1.97)$. Las preferencias menos aceptadas por los participantes, fueron el exclusionismo $(M=3.39 ; D E=2.07)$ y el asimilacionismo $(M=2.67 ; D E=1.87)($ Ver Tabla 1$)$.

Por otra parte, se observa que los participantes tienden a percibir bajos niveles de prejuicio afectivo, con una media de 2.03 $(D E=.73)$, en una escala de 5 puntos.

Además, los resultados muestran que el puntaje obtenido en la percepción de autoeficacia cultural es moderado, con una media de $3.58(D E=77)$, en una escala de 5 puntos, donde el puntaje más alto se presenta en la dimensión de autoeficacia para comprender otros estilos de vida $(M=3.71 ; D E=.92)$, seguida por la dimensión de autoeficacia para relacionarse con otras culturas $(M=3.64 ; D E=.86)$, y la 
dimensión de autoeficacia para procesar información de otras culturas $(M=3.35 ; D E=.84)$. Así también, se observa que los participantes perciben niveles adecuados de bienestar $(M=7.21 ; D E=1.85)$.

\section{Tabla 1}

Descriptivos de orientaciones de aculturación, prejuicio afectivo, autoeficacia cultural y bienestar

\begin{tabular}{lccc}
\hline & $n$ & $M$ & $S D$ \\
\hline Integracionismo & 426 & 5.09 & 1.74 \\
Individualismo & 426 & 5.49 & 1.64 \\
Asimilacionismo & 426 & 2.67 & 1.87 \\
Segregacionismo & 426 & 4.69 & 1.97 \\
Exclusionismo & 426 & 3.39 & 2.07 \\
Prejuicio afectivo & 426 & 2.03 & .73 \\
Autoeficacia cultural & 426 & 3.41 & .74 \\
Procesar información de otras culturas & 426 & 3.35 & .84 \\
Relacionarse con otras culturas & 426 & 3.64 & .86 \\
Comprender diferentes estilos de vida & 426 & 3.71 & .92 \\
Bienestar & 426 & 7.21 & 1.86 \\
\hline
\end{tabular}

\section{Perfiles aculturativos}

Los resultados de los análisis de conglomerados permitieron encontrar una solución de 3 grupos (Ver Tabla 2). El primero, está formado por el $45.5 \%$ de los participantes, y se caracteriza por un puntaje alto en individualismo, con una media de $6.18(D E=1.10)$ en una escala de 7 puntos, acompañado por un puntaje también alto en integracionismo $(M=5.88 ; D E=1.09)$. Además, este grupo presenta un puntaje moderado en segregacionismo $(M=4.67 ; D E=2.09)$, y una baja aceptación del exclusionismo $(M=1.94 ; D E=1.07)$ y asimilacionismo $(M=1.64$; $D E=.82)$. Estos puntajes sugieren un predominio de la tendencia a percibir a los inmigrantes como individuos con necesidades y metas personales, que solo de manera secundaria son decodificados como 
miembros de grupos socioculturales diferentes. La presencia de puntajes similares en integracionismo y segregación indicaría que cuando la pertenencia grupal es saliente, la aceptación del intercambio cultural con los inmigrantes podría aceptarse, pero restringida a ciertos ámbitos. En consecuencia, este grupo fue denominado "Individualista Tolerante".

El segundo grupo, que reúne al $30.3 \%$ de la muestra estudiada, presenta su puntaje más alto en individualismo, como una media de $6.05(D E=1.10)$ en una escala de 7 puntos, mientras que el segregacionismo $(M=5.76 ; D E=1.40)$, integracionismo $(M=5.62 ; D E=1.41)$ y exclusionismo $(M=5.57 ; D E=1.58)$ presentan medias similares, dando cuenta de un posicionamiento indiferenciado en cuanto a estas últimas variables. Por otra parte, se observa que el promedio obtenido por este grupo en asimilación $(M=4.17 ; D E=2.28)$ es mayor que la media de la muestra completa en esta variable, sin embargo, se encuentra en torno a la media teórica, lo cual nos indica una respuesta ambivalente o una indefinición respecto de la preferencia aculturativa. Estos resultados indican que la decodificación del otro como individuo es predominante también en este grupo y ante la cuestión del intercambio cultural, no presentan una actitud definida. Por esta razón, este grupo ha sido llamado "Individualista Indiferenciado".

El tercer grupo, que está compuesto por el $24.2 \%$ de los participantes, y presenta su media más alta en individualismo $(M=3.51$; $D E=1.47)$, seguida por exclusionismo $(M=3.38 ; D E=1.57)$, segregacionismo $(M=3.37 ; D E=1.52)$, integracionismo $(M=2.94$; $D E=1.27)$, y asimilacionismo $(M=2.73 ; D E=1.37)$. Si bien este grupo se caracteriza por la presencia de puntajes que en su mayoría se encuentran bajo el punto medio de la escala de 7 puntos, cabe señalar que la media más alta implica un muy bajo desacuerdo con la preferencia del individualismo, siendo las otras dimensiones aún más rechazadas que esta, dimensiones que suponen el reconocimiento de la pertenencia a categorías sociales. Si bien en este grupo se observan puntajes bajo la media teórica en todas las preferencias de aculturación, en conjunto configuran un perfil que se caracteriza por el desacuerdo al reconocimiento de los otros como miembros de una categoría cultural 
distinta. Cabe destacar que aun cuando el exclusionismo podría considerarse como el rechazo abierto a la expresividad de cualquier tipo de patrón cultural, incluso esta opción reconoce la pertenencia a algún exogrupo. Por lo tanto, este grupo ha sido definido como "Etnocentrista /Indiferenciado".

\section{Tabla 2}

Descriptivos de preferencias aculturativas por conglomerados para estudiantes de Educación Media

\begin{tabular}{lllll}
\hline \multicolumn{1}{c}{ Conglomerados } & & $n$ & $M$ & $S D$ \\
\hline & Integracionismo & 194 & 5.88 & 1.09 \\
& Individualismo & 194 & 6.18 & 1.10 \\
Individualista & Asimilacionismo & 194 & 1.64 & .82 \\
Tolerante & Segregacionismo & 194 & 4.67 & 2.09 \\
& Exclusionismo & 194 & 1.94 & 1.07 \\
\hline \multirow{3}{*}{ Individualista } & Integracionismo & 129 & 5.62 & 1.41 \\
Indiferenciado & Individualismo & 129 & 6.05 & 1.10 \\
& Asimilacionismo & 129 & 4.17 & 2.28 \\
& Segregacionismo & 129 & 5.76 & 1.40 \\
& Exclusionismo & 129 & 5.57 & 1.58 \\
\hline \multirow{3}{*}{$\begin{array}{l}\text { Indiferenciado } \\
\text { Etnocentrista / }\end{array}$} & Integracionismo & 103 & 2.94 & 1.27 \\
& Individualismo & 103 & 3.51 & 1.47 \\
& Asimilacionismo & 103 & 2.73 & 1.37 \\
& Segregacionismo & 103 & 3.37 & 1.52 \\
& Exclusionismo & 103 & 3.38 & 1.57 \\
\hline \multirow{2}{*}{} & & & &
\end{tabular}

\section{Prejuicio, autoeficacia cultural y bienestar de acuerdo a perfiles aculturativos}

Los resultados de los análisis de medias (ANOVA), muestran que existen diferencias significativas entre perfiles aculturativos en los puntajes obtenidos en prejuicio afectivo $(F(2,423)=37.62, p<0.001)$, 
donde las pruebas post hoc de Tukey indican que el grupo Individualista Tolerante $(M=1.73 ; D E=.51)$ presenta un puntaje menor que el perfil Individualista Indiferenciado $(M=2.24 ; D E=.82)$, y el perfil Etnocentrista Indiferenciado $(M=2.35 ; D E=.74)$. Esto sugiere que los participantes del grupo Individualista Tolerante presentan una actitud más positiva hacia el otro culturalmente distinto a diferencia de los otros dos perfiles, donde se observan medias más altas en las preferencias de aculturación, que suponen una valoración más bien negativa de las identidades culturales de los inmigrantes.

\section{Tabla 3}

Descriptivos y diferencias de medias de acuerdo a perfiles aculturativos en prejuicio afectivo, autoeficacia cultural y bienestar

\begin{tabular}{|c|c|c|c|c|c|c|c|}
\hline & & $n$ & $M$ & $D E$ & $F$ & $g l$ & $p$ \\
\hline \multirow{4}{*}{$\begin{array}{l}\text { Prejuicio } \\
\text { Afectivo }\end{array}$} & Individualista Tolerante & 194 & 1.73 & .51 & \multirow[t]{4}{*}{$37.618^{\mathrm{ab}}$} & \multirow[t]{4}{*}{423} & \multirow[t]{4}{*}{.000} \\
\hline & Individualista Indiferenciado & 129 & 2.24 & .82 & & & \\
\hline & Etnocentrista /Indiferenciado & 103 & 2.35 & .74 & & & \\
\hline & Total & 426 & 2.03 & .73 & & & \\
\hline \multirow{4}{*}{$\begin{array}{l}\text { Autoeficacia } \\
\text { cultural }\end{array}$} & Individualista Tolerante & 194 & 3.72 & .69 & \multirow[t]{4}{*}{$32.150^{\mathrm{bc}}$} & \multirow[t]{4}{*}{423} & \multirow[t]{4}{*}{.000} \\
\hline & Individualista Indiferenciado & 129 & 3.77 & .74 & & & \\
\hline & Etnocentrista /Indiferenciado & 103 & 3.08 & .77 & & & \\
\hline & Total & 426 & 3.41 & .74 & & & \\
\hline \multirow{4}{*}{$\begin{array}{l}\text { Procesar } \\
\text { información } \\
\text { de otras } \\
\text { culturas }\end{array}$} & Individualista Tolerante & 194 & 3.41 & .74 & \multirow[t]{4}{*}{$21.880^{\mathrm{bc}}$} & \multirow[t]{4}{*}{423} & \multirow[t]{4}{*}{.000} \\
\hline & Individualista Indiferenciado & 129 & 3.61 & .91 & & & \\
\hline & Etnocentrista /Indiferenciado & 103 & 2.92 & .78 & & & \\
\hline & Total & 426 & 3.35 & .84 & & & \\
\hline \multirow{4}{*}{$\begin{array}{l}\text { Relacionarse } \\
\text { con otras } \\
\text { culturas }\end{array}$} & Individualista Tolerante & 194 & 3.79 & .84 & \multirow[t]{4}{*}{$24.028^{\mathrm{bc}}$} & \multirow[t]{4}{*}{423} & \multirow[t]{4}{*}{.000} \\
\hline & Individualista Indiferenciado & 129 & 3.82 & .79 & & & \\
\hline & Etnocentrista /Indiferenciado & 103 & 3.16 & .80 & & & \\
\hline & Total & 426 & 3.64 & .86 & & & \\
\hline
\end{tabular}




\begin{tabular}{llcccccc}
\hline & & $n$ & $M$ & $D E$ & $F$ & $g l$ & $p$ \\
\hline Comprender & Individualista Tolerante & 194 & 3.92 & .86 & $31.187^{\mathrm{bc}}$ & 423 & .000 \\
diferentes & Individualista Indiferenciado & 129 & 3.84 & .82 & & & \\
estilos de & Etnocentrista /Indiferenciado & 103 & 3.12 & .92 & & & \\
vida & Total & 426 & 3.71 & .92 & & & \\
& Individualista Tolerante & 194 & 7.31 & 1.91 & $8.316^{\text {bc }}$ & 423 & .000 \\
Bienestar & Individualista Indiferenciado & 129 & 7.55 & 1.69 & & & \\
& Etnocentrista /Indiferenciado & 103 & 6.59 & 1.81 & & & \\
& Total & 426 & 7.21 & 1.86 & & & \\
\hline
\end{tabular}

Nota. Diferencia de medias pruebas post hoc de Tukey; ${ }^{\text {a }}$ diferencia de medias significativa entre Individualista Tolerante vs. Individualista Indiferenciado; ${ }^{b}$ : diferencias de medias significativas entre Individualista Tolerante vs Etnocentrista /Indiferenciado; c: diferencias de medias significativas entre Individualista Indiferenciado vs Etnocentrista/Indiferenciado.

También se observan diferencias estadísticamente significativas entre los perfiles en cuanto a su percepción de autoeficacia cultural $(F(2,423)=32.15, p<0.001)$. En específico, el perfil Etnocentrista Indiferenciado $(M=3.08 ; D E=.76)$ obtiene un puntaje significativamente más bajo que el grupo Individualista Tolerante $(M=3.72 ; D E=.79)$ e Individualista Indiferenciado $(M=3.77 ; D E=.74)$. Entre estos dos últimos grupos no existen diferencias significativas. En cada una de las dimensiones de la autoeficacia cultural se observa el mismo patrón, indicando por una parte que los participantes del grupo Etnocentrista Indiferenciado presentan una menor percepción de sí mismos como capaces de procesar información, relacionarse con otras culturas y comprender diferentes culturas. Así también, la no existencia de diferencias significativas entre los perfiles Individualistas Tolerantes e Indiferenciados sugiere que la vinculación con un otro diferente culturalmente, a partir de sus características personales y secundariamente como miembros de exogrupos, podría generar una mayor percepción de autoeficacia en quienes desarrollan estos tipos de patrones (Ver Tabla 3).

Asimismo, existen diferencias entre los grupos con respecto al bienestar percibido $(F(2,423)=32.15, p<0.001)$, donde el perfil 
Etnocentrista Indiferenciado $(M=6.60 ; D E=1.81)$ presenta un puntaje significativamente más bajo que el grupo Individualista Tolerante $(M=7.31 ; D E=1.90)$ e Individualista Indiferenciado $(M=7.55$, $D E=1.70)$. Entre estos dos últimos grupos no existen diferencias significativas. Lo anterior sugiere que las actitudes que tienden más a diferentes formas de rechazo de las identidades culturales del otro, se asocia a la presencia de mayores niveles de malestar (Ver tamańos del efecto en Tabla 4).

\section{Tabla 4}

Tamaños del efecto para diferencias de medias en prejuicio afectivo, autoeficacia cultural y bienestar, de acuerdo a perfiles aculturativos.

\begin{tabular}{lccc}
\hline Variable & $\begin{array}{l}\text { Individualista } \\
\text { Tolerante vs. } \\
\text { Individualista } \\
\text { Indiferenciado }\end{array}$ & $\begin{array}{l}\text { Individualista } \\
\text { Tolerante vs } \\
\text { Etnocentrista / } \\
\text { Indiferenciado }\end{array}$ & $\begin{array}{c}\text { Individualista } \\
\text { Indiferenciado } \\
\text { vs Etnocentrista } \\
\text { /Indiferenciado }\end{array}$ \\
\hline $\begin{array}{l}\text { Prejuicio Afectivo } \\
\text { Autoeficacia Cultural }\end{array}$ & .788 & 1.024 & $\begin{array}{l}\text { n.s. } \\
\text { Procesar información de otras } \\
\text { culturas }\end{array}$ \\
$\begin{array}{l}\text { Relacionarse con otras culturas } \\
\begin{array}{l}\text { Comprender diferentes estilos } \\
\text { de vida }\end{array}\end{array}$ & n.s. & .889 & .913 \\
\begin{tabular}{l} 
Bienestar \\
\hline
\end{tabular} & n.s. & .645 & .809 \\
\end{tabular}

Nota. Puntos de corte para el tamaño de efecto de la d de Cohen: .20 = pequeño; .50 = mediano; y .80 = grande (Cohen, 1988; Ellis, 2010).

\section{Discusión}

Este trabajo tuvo como objetivo identificar perfiles aculturativos en una muestra de adolescentes chilenos, y comprobar la existencia de diferencias entre ellos en prejuicio, autoeficacia cultural y bienestar. Los resultados para el total de los participantes muestran un predominio 
del individualismo como preferencia de aculturación, seguido por el integracionismo, el segregacionismo, el exclusionismo y el asimilacionismo. Este patrón, muy similar a los que ya han sido reportados en adolescentes chilenos por Mera-Lemp y Martínez-Zelaya, 2021, y así como en muestras de adultos chilenos (Sirlopú et al., 2015; Mera-Lemp et al., 2017; Mera-Lemp, Martínez-Zelaya, Bilbao \& Orellana, 2021). Esto sugiere que los adolescentes tienden a percibir a los inmigrantes latinoamericanos como individuos con características y objetivos personales, cuya participación en la comunidad receptora puede tener lugar mediante estrategias de movilidad social individual (Bourhis et al., 1997; Tajfel \& Turner, 1972). Este predominio del individualismo es consistente con lo planteado por Santos, Michel, Varnum y Grossmann (2017) con respecto a la relación entre el aumento de prácticas y valores individualistas en el mundo durante la última década y el crecimiento económico.

Así también, este resultado da cuenta de la aceptación de que los inmigrantes latinoamericanos adopten algunos elementos de la cultura de la sociedad mayoritaria, pero manteniendo sus valores y prácticas culturales en la esfera de lo privado, lo que sugiere cierto grado de percepción de amenaza simbólica (Stephan et al., 2005; Wlodarczyk et al., 2014).

Por otra parte, se observa una baja percepción de prejuicio afectivo hacia los inmigrantes latinoamericanos por parte de los adolescentes, lo que también ha sido observado en otros estudios con muestras de adolescentes chilenos (Mera-Lemp, Bilbao \& Basabe, 2020). En cuanto a la percepción de autoeficacia cultural general, los adolescentes se sienten moderadamente capaces de interactuar exitosamente con miembros de otros grupos culturales. La dimensión de autoeficacia para comprender otros estilos de vida obtiene el puntaje mayor, seguido por la dimensión de autoeficacia para relacionarse con otras culturas, y la dimensión de autoeficacia para procesar información de otras culturas, que obtuvo el menor puntaje, similar a lo observado en otros estudios en adolescentes chilenos (Mera-Lemp, Bilbao \& Basabe, 2020). Asimismo, el nivel de bienestar de los participantes es adecuado. 
El análisis de conglomerados muestra que el $45.5 \%$ de los participantes corresponden al grupo denominado "Individualista Tolerante", donde el individualismo aculturativo es matizado por la lectura del otro como miembro de un grupo cultural diferente, que tiene como consecuencia la aceptación del intercambio cultural restringido a ciertos ámbitos (Bourhis et al., 1997). Este posicionamiento parece ser la forma de aproximación más positiva en términos del tipo de relación que los adolescentes pudieran establecer con los inmigrantes, especialmente en el contexto escolar chileno, ya que no supone un rechazo abierto a la identidad cultural del otro, ni la presencia de un conflicto explicito (Berry, Phinney, Sam \& Vedder, 2006; Mera-Lemp \& Martínez-Zelaya, 2021). Por otra parte, este cluster es muy similar al encontrado en un estudio anterior con adultos chilenos (Mera et al., 2017), lo que sugeriría la presencia de un patrón cultural que podría estar consolidándose, fenómeno ya estudiado en Chile hace dos décadas (PNUD, 2002), y que debería de seguir siendo investigado en mayor profundidad en futuros estudios.

El grupo "Individualista Indiferenciado" (30.3\%), muestra un predominio del individualismo, acompañado por una baja diferenciación entre las demás orientaciones de aculturación, salvo la asimilación, que si bien se encuentra en el punto medio de la escala, es más alto que en el resto de los grupos, así como también en contraste con resultados de estudios anteriores en adultos chilenos (Mera et al., 2017; Mera-Lemp, Martínez-Zelaya, Bilbao \& Orellana, 2021). Es importante recordar, que el individualismo supone la decodificación del otro en tanto individuo con características y metas personales, y por lo tanto, supondría la emergencia de actitudes y conductas interpersonales (Tajfel \& Turner, 1979). Los puntajes obtenidos en las variables que suponen la categorización del otro como un miembro de un grupo cultural son similares, lo que podría estar indicando que la diferencia cultural no es relevante para estos participantes, aun cuando las identidades culturales de los miembros del exogrupo no son rechazadas. En este sentido, este tipo de posicionamiento permitiría que la relación establecida el otro diferente se basa en las características personales sin presentar un 
rechazo explícito a sus pertenecías grupales. Esta indiferenciación también podría deberse a los procesos construcción de identidad propios de la adolescencia, y en específico, al desarrollo de la Identidad Étnica en esta etapa del ciclo vital (Berry et al., 2006; Phinney \& Baldelomar, 2011).

El tercer grupo, denominado "Etnocentrista Indiferenciado", presenta una mayor saliencia de la pertenencia grupal de las personas inmigrantes $y$, aunque sus puntajes en las diversas alternativas de aculturación son similares, dan cuenta de una mayor tendencia a la percepción negativa de las identidades étnicas de los miembros del exogrupo. Este grupo corresponde al 24.2\% de los participantes. Aunque se trata de un grupo de menor tamaño, el posicionamiento de este clúster respecto del aceptación y valoración de la cultura del otro, el cual puede tener consecuencias en el bienestar de los adolescentes inmigrantes. En este sentido, si bien todos los puntajes en este clúster están bajo la media teórica, esta aproximación a los estudiantes inmigrantes podría poner en riesgo una convivencia escolar armónica a futuro, dada la dificultad para comprender los recursos culturales del otro, que pueden ser fuente de identidad y bienestar en el adolescente inmigrante (Berry, Phinney, Sam \& Vedder, 2006; Mera-Lemp, Martínez-Zelaya, Orellana \& Smith-Castro, 2020). Al igual que en el grupo Individualista Indiferenciado, en este clúster no se observa un claro, lo que también podría atribuirse a los procesos de elaboración de sus propias identidades culturales durante la adolescencia (Berry et al, 2006; Phinney \& Baldelomar, 2011).

El segundo objetivo de este estudio fue el comparar las medias de prejuicio, autoeficacia cultural y bienestar entre los distintos conglomerados. Los resultados confirman la hipótesis que seńalaba que los participantes que presentaran una mayor tendencia hacia el integracionismo e individualismo, presentarían también menor prejuicio (Briones et al., 2012; Brown \& Zagefka, 2011; Navas et al., 2006; Navas \& Rojas, 2010; Zagefka et al., 2012). Así, el perfil "Individualista Tolerante" presento un puntaje significativamente menor en prejuicio que el grupo "Etnocentrista Indiferenciado", y que el grupo 
"Individualista Indiferenciado". Esto indicaría que el perfil "Individualista Tolerante" estaría desplegando una mirada más positiva hacia los inmigrantes, y no los decodifica como una amenaza como los otros dos (Bourhis et al., 1997; Navas et al., 2006). Este hallazgo, reafirma la necesidad de incorporar una perspectiva intercultural en los contextos escolares, que incluya el trabajo acerca de las creencias acerca de los otros culturalmente distintos, promoviendo el establecimiento de relaciones intergrupales positivas.

Además, también se confirma la hipótesis que sugería que los participantes que mostraran una mayor tendencia hacia el integracionismo e individualismo presentarían mayores niveles de autoeficacia cultural (Li \& Gasser, 2005; Rania et al., 2012; Rockstuhl \& Ng, 2008; Rodenborg y Boisen, 2013; Spencer-Rodgers \& McGovern, 2002; Ting-Toomey, 2009). El grupo "Etnocentrista Indiferenciado", presenta un puntaje significativamente menor que los demás grupos en la percepción de competencia para relacionarse con miembros de otros grupos culturales, mostrando una menor capacidad para procesar información de otras culturas, relacionarse con otras culturas, y comprender diferentes estilos de vida. El desarrollo de las competencias interculturales requiere de emociones positivas hacia el exogrupo, que propicien la construcción de expectativas positivas ante el contacto, así como el reconocimiento y valoración de la diferencia cultural. Esto explicaría los menores niveles de autoeficacia cultural percibida por este perfil.

Así también el grupo "Etnocentrista Indiferenciado" presentó una menor percepción de bienestar que los otros grupos, los cuales presentan altos puntajes en individualismo. Esto es coherente con estudios antecedentes que sugerían que altos puntajes en integración e individualismo se relacionarían con mayores niveles de bienestar (Mera et al., 2017; Migliorini, 2016).

En conjunto estos resultados apoyan la evidencia de estudios previos en muestras chilenas (Mera et al., 2017; Mera-Lemp, et al., 2021; Mera-Lemp \& Martínez-Zelaya, 2021), donde el individualismo parece ser un elemento cultural que enmarca las relaciones con los otros. Altos puntajes en ello se asocian a mejor funcionamiento 
psicológico (Martínez-Zelaya, Mera-Lemp y Bilbao, 2021; MeraLemp, et al., 2021) y social (Mera et al., 2017), además de relacionarse con una mayor percepción de competencia para entablar relaciones interculturales, aun cuando pueden diferir en los niveles de prejuicio entre perfiles altos en individualismo.

Como principales limitaciones de este estudio, cabe señalar la utilización de una muestra de conveniencia en el marco de un diseño transversal, que no hace posible observar la relación entre las variables estudiadas a lo largo del tiempo. Otra limitante del uso de una muestra por conveniencia es la imposibilidad de representatividad de dicha muestra respecto de la población. Futuros estudios deberían indagar cualitativamente en los perfiles encontrados para dotarlos de profundidad de contenido y posibles relaciones más complejas de explicar con una metodología transversal.

No obstante, consideramos que los resultados obtenidos resaltan la importancia de profundizar en las posibles implicancias del individualismo aculturativo como forma de relacionarse con otros diferentes, que ya había sido observado en estudios previos con muestras adolescentes (Mera-Lemp \& Martínez-Zelaya, 2021) y de adultos chilenos (Mera et al, 2017; Martínez-Zelaya, et al., 2020; Mera-Lemp, MartínezZelaya, Bilbao \& Orellana, 2021). Esto contrasta con los resultados de estudios anteriores realizados con muestras de adolescentes y adultos inmigrantes latinoamericanos en Chile, donde destaca la presencia preponderante del Integracionismo. Este desacuerdo eventualmente generar dinámicas relacionales problemáticas de baja de intensidad (Bourhis, et al., 1997).

Así también, queremos destacar la relevancia del desarrollo temprano de competencias interculturales, especialmente en el contexto de sociedades que, como la nuestra, presentan un tejido social cada vez más complejo y diverso a propósito de la inmigración. Estas competencias cuentan con la ventaja de que pueden ser entrenadas, por ejemplo, en la escuela, facilitando una mejor convivencia entre estudiantes de distintas procedencias. Por último, pensamos que este trabajo constituye un aporte al avance de una línea de investigación que no ha 
sido suficientemente desarrollada en nuestro país, y cuya relevancia está dada por la necesidad de comprender los modos en que los adolescentes chilenos están vinculándose con los nuevos escenarios de diversidad cultural producidos por la inmigración.

\section{Referencias}

Aberson, C. L. (2015). Positive intergroup contact, negative intergroup contact, and threat as predictors of cognitive and affective dimensions of prejudice. Group Processes y Intergroup Relations, 18(6), 743-760.

Barnett, S. W. (2008). Preschool education and its lasting effects: Research and policy implications. Boulder and Tempe: Education and the Public Interest Center y Education Policy Research Unit.

Barrios-Valenzuela, A. y Palou-Julián, B. (2014). Educación intercultural en Chile: la integración del alumnado extranjero en el sistema escolar. Revista Educación y Educadores, 17(3), 405-426

Berry, J. W., Phinney, J. S., Sam, D. L., and Vedder, P. (2006). Immigrant youth: acculturation, identity, and adaptation. Appl. Psychol. 55, 303-332. https://doi.org/10.1111/j.1464-0597.2006.00256.x

Bhawuk, D., Sakuda, K. H., y Munusamy, V. P. (2008). Intercultural Competence Development and Triple-Loop Cultural Learning: Toward a theory of intercultural sensitivity. In S. Ang y L. Van Dyne (Eds.), Handbook of Cultural Intelligence: Theory, measurement, and implications (pp. 342-355). Armonk, NY: M.E. Sharpe.

Bourhis, R. Y., Moïse, L. C., Perreault, S., y Senécal, S. (1997). Towards an Interactive Acculturation Model: A Social Psychological Approach. International Journal of Psychology, 32(6), 369-386. https://doi.org/10.1080/002075997400629

Calzada, E. J., Huang, K. Y., Hernandez, M., Soriano, E., Acra, C. F., Dawson-McClure, S., ... y Brotman, L. (2014). Family and teacher characteristics as predictors of parent involvement in 
education during early childhood among Afro-Caribbean and Latino immigrant families. Urban Education, 50(7), 870-896.

Carter, P. (2006). Straddling Boundaries: Identity, Culture, and School. Sociology of Education, 79(3), 304-328.

Chen, G. M., y Starosta, W. J. (1998-9). A review of the concept of intercultural awareness. Human Communication, 2, 27-54.

Cohen, J. (1988). Statistical Power Analysis for the Behavioral Sciences. New York: Lawrence Erlbaum Associates.

Cortázar, A. (2015). Long-term effects of public early childhood education on academic achievement in Chile. Early Childhood Research Quarterly, 32, 13-22.

De la Torre, P. (2011). La migración vivenciada en el espacio escolar: la experiencia de alumnado extranjero en una escuela pública de Santiago. Paulo Freire. Revista de Pedagogía Crítica, 10, 9, 113-126.

Dovidio, J. F., Hewstone, M., Glick, P., y Esses, V. M. (2010). Prejudice, stereotyping and discrimination: Theoretical and empirical overview. The SAGE handbook of prejudice, stereotyping and discrimination, 3-29.

Earley, P. C., y Ang, S. (2003). Cultural intelligence: Individual interactions across cultures. Stanford University Press.

Ellis, P. (2010). The essential guide to effect sizes: statistical power, metaanalysis, and the interpretation of research results. Cambridge, Reino Unido: Cambridge University Press.

Frenzel, A. C., Becker-Kurz, B., Pekrun, R., Goetz, T., y Lüdtke, O. (2017). Emotion transmission in the classroom revisited: A reciprocal effects model of teacher and student enjoyment. Journal of Educational Psychology, 110(5), 628-639.

Giovannini, D., y Vezzali, L. (2011). Contact with Immigrant Parents as a Predictor of Teachers' Attitudes and Acculturation Orientations toward Immigrant Children. International Journal about Parents in Education, 5(2), 65-76.

Hagenauer, G., Hascher, T., y Volet, S. E. (2015). Teacher emotions in the classroom: associations with students' engagement, classroom 
discipline and the interpersonal teacher-student relationship. European Journal of Psychology of Education, 30(4), 385-403.

Hein, K. (2012). Migración y transición: hijos de inmigrantes de origen latinoamericano en su transición de la escuela al trabajo en Chile. Si Somos Americanos, 12(1), 101-126.

Hernández, A. (2016). El currículo en contextos de estudiantes migrantes: Las complejidades del desarrollo curricular desde la perspectiva de los docentes de aula. Estudios Pedagógicos, 42(2), 151-169.

Horenczyk, G., y Tatar, M. (2002). Teachers' attitudes toward multiculturalism and their perceptions of the school organizational culture. Teaching and Teacher Education, 18(4), 435-445.

Instituto Nacional de Estadísticas (2018). Resultados Censo 2017. Recuperado de https://redatamine.ine.cl/redbin/RpWebEngine. exe/Portal?BASE=CENSO_2017ylang=esp

Instituto Nacional de Estadísticas (INE) y Departamento de Extranjería y Migración (DEM), (2020). Estimación de personas extranjeras residentes habituales en Chile al 31 de diciembre 2019. Santiago de Chile: INE, recuperado de https://www. ine.cl/docs/default-source/demografia-y-migracion/publicaciones-y-anuarios/migraci $\% \mathrm{C} 3 \% \mathrm{~B} 3 \mathrm{n}$-internacional/ estimaci\%C3\%B3n-poblaci\%C3\%B3n-extranjera-en-chile-2018/ estimaci $\% \mathrm{C} 3 \% \mathrm{~B} 3 n$-poblaci $\% \mathrm{C} 3 \% \mathrm{~B} 3 n$-extranjera-en-chile2019-metodolog\%C3\%ADa.pdf?sfvrsn=5b145256_6

Kao, G. y Thompson, J. S. (2003). Racial and Ethnic Stratification in Educational Achievement and Attainment. Annual Review of Sociology, 29, 417-442.

Keltner, D., y Haidt, J. (1999). Social functions of emotions at four levels of analysis. Cognition y Emotion, 13(5), 505-521.

Kemper, T. D. (1984). Power, status, and emotions: A sociological contribution to a psychophysiological domain. En: Ekman, P., y Scherer, K. R. (Eds.), Approaches to emotion. USA: Erlbaum Associates, pp.377-378. 
Leung, K., Ang, S., y Tan, M. L. (2014). Intercultural competence. Annu. Rev. Organ. Psychol. Organ. Behav., 1(1), 489-519.

Mera, M.J., Martínez-Zelaya, G., Bilbao, MA. y Garrido, A. (2017)

Chilenos ante la inmigración: un estudio de las relaciones entre orientaciones de aculturación, percepción de amenaza y bienestar social en el Gran Concepción. Universitas Psychologica, 16(5), 1-14.

Mera-Lemp, M.J., Bilbao, M. y Basabe, N. (2020). School satisfaction in immigrant and Chilean students: the role of prejudice and cultural self-efficacy. Frontiers in Psychology, 11, 3393. https:// doi.org/10.3389/fpsyg.2020.613585

Mera-Lemp, M.J., Martínez-Zelaya, G., Bilbao, M. y Orellana, A. (2021) Chilean host society members and Latin-American immigrants: intergroup relations perceptions, acculturation orientations and well-being. En V. Smith-Castro, D. Sirlopú, A. Eller y H. Cakal, Psychological perspectives on Intra-Regional Migration in Latin America (pp. 19-50). Washington, D.C., USA: APA Books.

Mera-Lemp, M.J., y Martínez-Zelaya, G. (2021) Relaciones intergrupales en la escuela: cercanía social, prejuicio y aculturación en estudiantes inmigrantes latinoamericanos y chilenos. Inclusão Social, 13(2), 220-233, http://revista.ibict.br/inclusao/issue/ view/300

Ministerio de Educación de Chile (2018). Estadisticas de la Educación 2017, Publicación diciembre 2018. Santiago de Chile: Centro de Estudios MINEDUC. Recuperado de https://centroestudios.mineduc.cl/wp-content/uploads/sites/100/2018/12/ ANUARIO-MINEDUC_VERSION-BAJA.pdf

Mondaca C., Rivera P., y Gajardo, Y. (2014). Educación parvularia e inclusión en el norte de Chile: Formando pequeños chilenos en las aulas de Tarapacá. Alpha (Osorno), (39), 251-266.

Naudon, P. (2016). Mujeres migrantes en Chile: significaciones sobre su rol de madre y la crianza de hijos. Revista Rumbos TS, (14), 99-112. 
Navas, L., Holgado, F. P., y Sánchez, A. (2009). Predicción de los estereotipos académicos ante los estudiantes inmigrantes. Horizontes educacionales, $14(2)$.

Navas, L. y Sánchez, A. (2010). Actitudes de los Estudiantes de Pedagogía de las Regiones del Bío Bío y la Araucanía de Chile hacia la Presencia de Niños Inmigrantes en la Escuela: Análisis Diferenciales. Psykhe, 19(1), 47-60.

Navas, M., y Rojas, A. J. (2010). Aplicación del Modelo Ampliado de Aculturación Relativa (MAAR) a nuevos colectivos de inmigrantes en Andalucía: ecuatorianos y rumanos. Sevilla: Junta de Andalucía, Consejería de Empleo.

Pajares, F. (2007) Culturalizing educational psychology. In F. Salili y R. Hoosain (Eds.), Culture, motivation and learning: $A$ multiculturalperspective (pp. 19-42). Charlotte, NC: Information Age Publishing.

Pavez, I. (2012) Inmigración y racismo: experiencias de la niñez peruana en Santiago de Chile. Si Somos Americanos, 12(1), 75-99.

Pettigrew, T. F., y Meertens, R. W. (1995). Subtle and blatant prejudice in Western Europe. European Journal Of Social Psychology, 25(1), 57-75.

Pettigrew, T. F., y Tropp, L. R. (2000). Does intergroup contact reduce prejudice? Recent meta-analytic findings. Reducing prejudice and discrimination, 93, 114.

Phinney, J. S., y Baldelomar, O. A. (2011). Identity development in multiple cultural contexts. In L. A. Jensen (Ed.), Bridging cultural and developmental approaches to psychology: New syntheses in theory, research and policy (pp. 161-186). New York: Oxford University Press.

Pizarro, P. y Espinoza, V. (2016). ¿Calidad en la formación inicial docente? Análisis de los nuevos estándares de la educación de párvulos en Chile. Perspectiva Educacional, 55(1), 152-167.

Poblete, R. y Galaz, C. (2017). Aperturas y cierres para la inclusión educativa de niños/as migrantes en Chile. Estudios Pedagógicos, 3, 239-257. 
Rodenborg, N. A., y Boisen, L. A. (2013). Aversive racism and intergroup contact theories: Cultural competence in a segregated world. Journal of Social Work Education, 49(4), 564-579.

Riedemann, A., y Stefoni, C. (2014). Sobre el racismo, su negación, y las consecuencias para una educación anti-racista en la enseñanza secundaria chilena. Polis, 14(42), 191-216.

Salas, N., Castillo, D., San Martín, C., Kong, F., Thayer, L.E., y Huepe, D. (2017) Inmigración en la escuela: caracterización del prejuicio hacia escolares migrantes en Chile. Universitas Psychologica, 16(5), 1-15.

Sánchez, E. (2017). Desafíos de la institucionalidad en la educación parvularia en chile. Análisis a partir de la reforma educacional 2014-2018. Estado, Gobierno y Gestión Pública, (30), 45-68.

Sánchez, E., Díaz, A., Mondaca, C., y Mamani, J. C. (2018). Formación inicial docente, prácticas pedagógicas y competencias interculturales de los estudiantes de carreras de pedagogía de la Universidad de Tarapacá, Norte De Chile. Diálogo Andino, (57), 21-38.

Sanhueza, S. V., Friz Carrillo, M. C., y Quintriqueo Millán, S. (2014). Estudio exploratorio sobre las actitudes y comportamiento del profesorado de Chile en contextos de escolarización de alumnado inmigrante. Revista electrónica de investigación educativa, 16(3), 148-162.

Seeberg, V., y Minick, T. (2012). Enhancing cross-cultural competence in multicultural teacher education: Transformation in global learning. International Journal of Multicultural Education, 14(3).

Siqués, C.; Vila, I. y Perera, S. (2009). Percepciones y actitudes del alumnado extranjero y del profesorado: un estudio empírico en las aulas de acogida de Cataluña. Electronic Journal of Research in Educational Psychology, 17, 7 (1), 103-132.

Sirlopú, D., y Van Oudenhoven, J. P. (2013). Is multiculturalism a viable path in Chile? Intergroup and acculturative perspectives on Chilean society and Peruvian immigrants. International Journal of Intercultural Relations, 37(6), 739-749. 
Spencer-Rodgers, J., y McGovern, T. (2002). Attitudes toward the culturally different: The role of intercultural communication barriers, affective responses, consensual stereotypes, and perceived threat. International Journal of Intercultural Relations, 26(6), 609-631.

Stephan, W., Renfro, C., Esses, V., Stephan, C., y Martin, T. (2005). The effects of feeling threatened on attitudes toward immigrants. International Journal of Intercultural Relations, 29(1), 1-19.

SubsecretaríadeEducación Parvularia (2018) Basescurriculares Educación Parvularia. Santiago de Chile: MINEDUC. Recuperado de https://parvularia.mineduc.cl/wp-content/uploads/ sites/34/2018/03/Bases_Curriculares_Ed_Parvularia_2018.pdf

Tajfel, H., y Turner, J. (1979). An integrative theory of intergroup conflict. In W. G. Austin, y S. Worchel (Eds.), The social psychology of intergroup relations (pp. 33-47). Monterey: CA: Brooks/Cole.

Tijoux, M. E. (2013). Las escuelas de la inmigración en la ciudad de Santiago: Elementos para una educación contra el racismo. Polis, 12(35), 287-307.

Ting-Toomey, S. (2009). Intercultural conflict competence as a facet of intercultural competence development: Multiple conceptual approaches. In D. Deardorff (Ed.), The Sage handbook of intercultural competence (pp. 100-120). California, USA: SAGE Publications.

Tropp, L. R., y Pettigrew, T. F. (2005). Relationships between intergroup contact and prejudice among minority and majority status groups. Psychological Science, 16(12), 951-95

Tyler, K. M., Boykin, A. W., y Walton, T. R. (2006). Cultural considerations in teachers' perceptions of student classroom behavior and achievement. Teaching and Teacher Education, 22, 998-1005. https://doi.org/10.1016/j.tate.2006.04.017

Vecina, C. (2006). La violencia simbólica en la interacción entre docentes y alumnos inmigrantes. Revista Aposta de Ciencias Sociales, 24, 1-17. 
Vedder, P., Wenink, E., y van Geel, M. (2016). Explaining negative outgroup attitudes between native Dutch and Muslim youth in The Netherlands using the Integrated Threat Theory. International Journal of Intercultural Relations, 53, 54-64.

Velasco, K., Verkuyten, M., Weesie, J., y Poppe, E. (2008). Prejudice towards Muslims in the Netherlands: Testing integrated threat theory. British Journal of Social Psychology, 47(4), 667-685.

Verkuyten, M. y Thijs, J. (2002) School Satisfaction of Elementary School Children: The Role of Performance, Peer Relations, Ethnicity and Gender. Social Indicators Research, 59, 203-228.

Ward, C., y Masgoret, A. M. (2008). Attitudes toward immigrants, immigration, and multiculturalism in New Zealand: A social psychological analysis. International Migration Review, 42(1), 227-248.

Wong, C., Eccles, J. y Sameroff, A. (2003) The Influence of Ethnic Discrimination and Ethnic Identification on African American Adolescents' School and Socioemotional Adjustment. Journal of Personality, 71(6), 1198-1232.

Yzerbyt, V., y Demoulin, S. (2010). Intergroup relations. Handbook of Social Psychology, 2, 1024-1083.

Recibido: 14 de enero, 2020

Revisado: 10 de diciembre, 2020

Aceptado: 6 de abril, 2021 